Claremont Colleges

Scholarship@ Claremont

All HMC Faculty Publications and Research

HMC Faculty Scholarship

$1-1-1988$

\title{
Nonnegative Solutions for a Class of Nonpositone Problems
}

\author{
Alfonso Castro \\ Harvey Mudd College \\ Ratnasingham Shivaji \\ Mississippi State University
}

\section{Recommended Citation}

A. Castro and R. Shivaji. "Non-negative solutions for a class of non-positone problems," Proc. Royal Soc. Edinburgh, 108A, (1988), pp. 291-302.

This Article is brought to you for free and open access by the HMC Faculty Scholarship at Scholarship @ Claremont. It has been accepted for inclusion in All HMC Faculty Publications and Research by an authorized administrator of Scholarship @ Claremont. For more information, please contact scholarship@cuc.claremont.edu. 


\title{
Non-negative solutions for a class of non-positone problems
}

\author{
Alfonso Castro \\ Department of Mathematics, North Texas State University, \\ Denton, TX 76203-5116, U.S.A. \\ and \\ R. Shivaji \\ Department of Mathematics, Mississippi State University, \\ Mississippi State, MS 39762, U.S.A. \\ (MS received 12 February 1987. Revised MS received 11 September 1987)
}

\section{Synopsis}

In the recent past many results have been established on non-negative solutions to boundary value problems of the form

$$
\begin{aligned}
-u^{\prime \prime}(x) & =\lambda f(u(x)) ; \quad 0<x<1, \\
u(0) & =0=u(1),
\end{aligned}
$$

where $\lambda>0, f(0)>0$ (positone problems). In this paper we consider the impact on the non-negative solutions when $f(0)<0$. We find that we need $f(u)$ to be convex to guarantee uniqueness of positive solutions, and $f(u)$ to be appropriately concave for multiple positive solutions. This is in contrast to the case of positone problems, where the roles of convexity and concavity were interchanged to obtain similar results. We further establish the existence of non-negative solutions with interior zeros, which did not exist in positone problems.

\section{Introduction}

Consider the two point boundary value problem

$$
\begin{aligned}
-u^{\prime \prime}(x) & =\lambda f(u(x)) ; \quad x \in(0,1), \\
u(0) & =0=u(1),
\end{aligned}
$$

where $\lambda>0$ is a constant and $f \in \mathbb{C}^{2}$ satisfies

$$
f(0)<0 .
$$

Firstly, note that any solution $u(x)$ of (1.1)-(1.2) is symmetric about any point $x_{0} \in(0,1)$ such that $u^{\prime}\left(x_{0}\right)=0$. Then by $(1.3)$ it is possible that a non-negative solution $u(x)$ of $(1.1)-(1.2)$ may have interior zeros in $(0,1)$. (This was not the case when $f(0)>0$, where if $u(x)$ is a non-negative solution of $(1.1)-(1.2)$, then $u(x)>0$ on $(0,1)$.) We will distinguish those solutions which do not have interior zeros by referring to them as positive solutions. We shall consider three distinct cases:

$$
\begin{aligned}
& \text { Case (A). } f^{\prime \prime}(s)>0 \text { for } s>0, \\
& \text { Case (B). } f^{\prime \prime}(s)<0 \text { for } s>0,
\end{aligned}
$$


Case (C). There exists some $s_{0}$ such that $f^{\prime \prime}(s)<0$ for $0<s<s_{0}$ and $f^{\prime \prime}(s)>0$ for $s>s_{0}$.

Our main results are:

THEOREM 1.1. Let $f(0)<0, f^{\prime}(s)>0$ for $s>0$ and $\beta, \theta$ be positive real numbers that satisfy $f(\beta)=0, F(\theta)=0$, respectively, where $F(s)=\int_{0}^{s} f(t) d t$.

Case (A). If $f^{\prime \prime}(s)>0$ for $s>0$ and $\lim _{s \rightarrow+\infty} f(s) / s=+\infty$, then there exist $\lambda^{*}>0$ such that (1.1)-(1.2) have a unique positive solution for $0<\lambda \leqq \lambda^{*}$ and have no positive solutions for $\lambda>\lambda^{*}$. Also denoting by $\rho_{\lambda}$ the supremum norm of the positive solution, $\rho_{\lambda}$ increases as $\lambda$ decreases, and in particular, $\rho_{\lambda^{*}}=\theta$, $\lim _{\lambda \rightarrow 0} \rho_{\lambda}=+\infty$. (See Fig. 1.1.)

Case (B). If $f^{\prime \prime}(s)<0$ for $s>0, \lim _{s \rightarrow+\infty} f(s)=M$ where $0<M \leqq+\infty$, $\lim _{s \rightarrow+\infty} s f^{\prime}(s)=0$ and $(f(\theta) / \theta)<f^{\prime}(\theta)$, then there exist $\lambda^{*}, \mu_{1}$ such that $0<\mu_{1}<\lambda^{*}$ and (1.1)-(1.2) have no positive solutions for $0<\lambda<\mu_{1}$ and at least one positive solution for $\lambda \geqq \mu_{1}$. Further (1.1)-(1.2) have at least two positive solutions for $\mu_{1}<\lambda \leqq \lambda^{*}$ and there exists $\mu_{2} \geqq \lambda^{*}$ such that (1.1)-(1.2) have a unique positive solution for $\lambda>\mu_{2}$. Also, $\rho_{\lambda^{*}}=\theta$ and $\lim _{\lambda \rightarrow+\infty} \rho_{\lambda}=+\infty$. (See Fig. 1.2.)
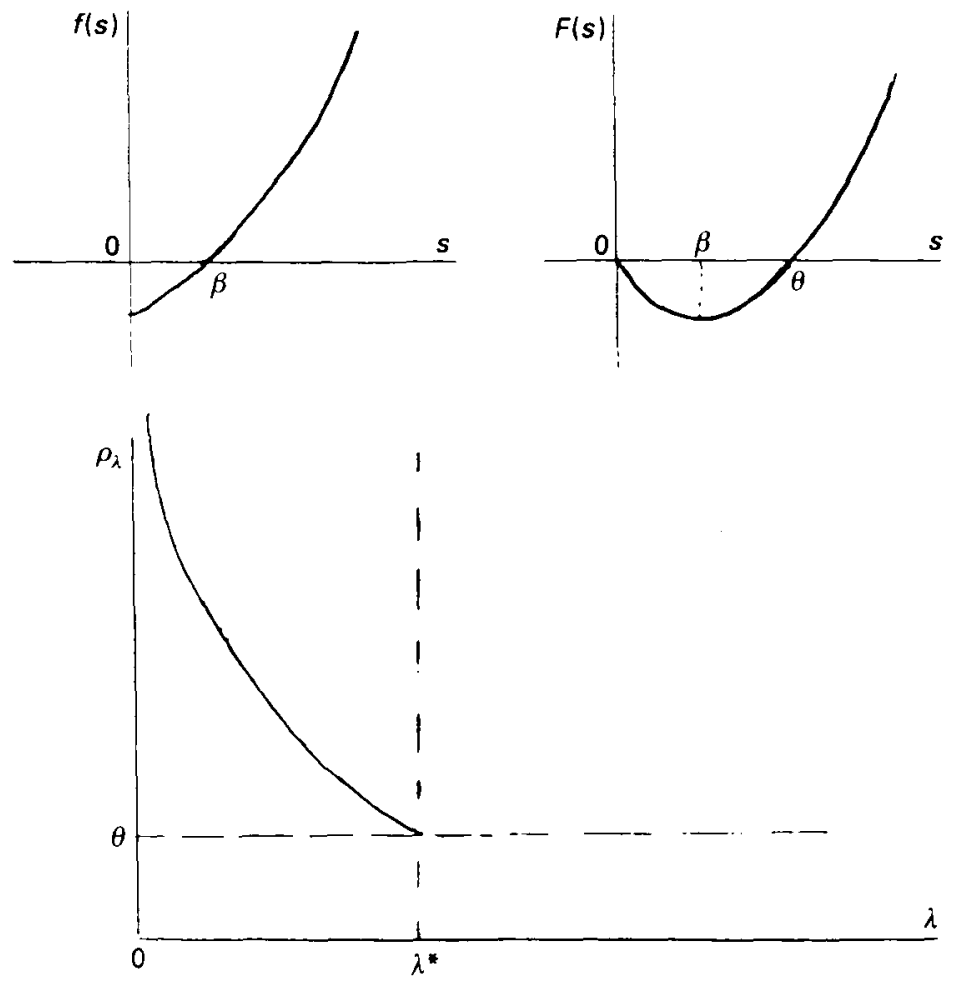

Figure 1.1. Bifurcation diagram (Theorem 1.1, Case (A)). 
Non-negative solutions for a class of non-positone problems
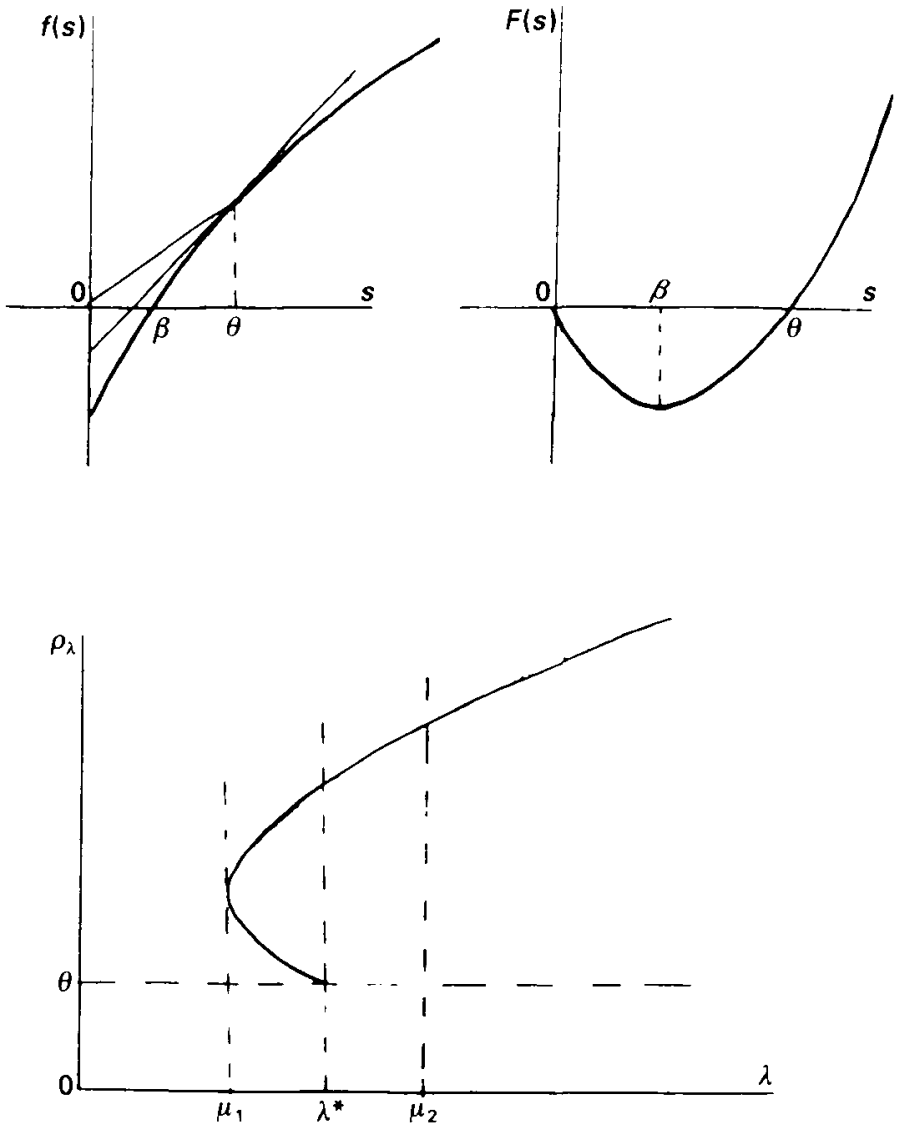

Figure 1.2. Bifurcation diagram (Theorem 1.1, Case (B)).

Case (C). If $f^{\prime \prime}(s)<0$ for $s \in\left(0, s_{0}\right)$ with $s_{0}>\theta, f^{\prime \prime}(s)>0$ for $s>s_{0},(f(\theta) / \theta)<$ $f^{\prime}(\theta)$, and if there exists $\sigma>\theta$ such that $H(\sigma)=F(\sigma)-(\sigma / 2) f(\sigma)>0$, $\lim _{s \rightarrow+\infty}(f(s) / s)=+\infty$ and $\lim _{s \rightarrow+\infty} f(s)-\lim _{s \rightarrow+\infty} s f^{\prime}(s)<0$, then there exist $\lambda^{*}, \lambda_{1}, \lambda_{2}$ such that $0<\lambda_{1}<\lambda^{*} \leqq \lambda_{2}$ and (1.1)-(1.2) have a unique positive solution for $0<\lambda<\lambda_{1}$ and no positive solutions for $\lambda>\lambda_{2}$. Further, there exists a range for $\lambda$ in $\left(\lambda_{1}, \lambda^{*}\right)$ in which (1.1)-(1.2) have at least three positive solutions and if $\lambda_{2}>\lambda^{*}$ then (1.1)-(1.2) have at least two positive solutions for $\lambda \in\left[\lambda^{*}, \lambda_{2}\right)$. Also $\rho_{\lambda^{*}}=\theta$ and $\lim _{\lambda \rightarrow 0} \rho_{\lambda}=+\infty$. (See Fig. 1.3.)

Lemma 1.2. Let $\alpha=\theta^{2} /[-F(\beta)]$. Then $2 \alpha \leqq \lambda^{*} \leqq 8 \alpha$.

LEMMA 1.3. If $u_{1}(x)$ and $u_{2}(x)$ are distinct positive solutions of (1.1)-(1.3), then $\left\{x \in(0,1) / u_{1}(x)=u_{2}(x)\right\}$ is the empty set.

THEOREM 1.4. Let the hypotheses of Theorem 1.1 hold. Then in each of the cases (A), (B), or (C), given $n$ a positive integer, (1.1)-(1.2) have a non-negative solution with $n$ interior zeros if and only if $\lambda=(n+1)^{2} \lambda^{*}$. Further, such a solution is unique. 

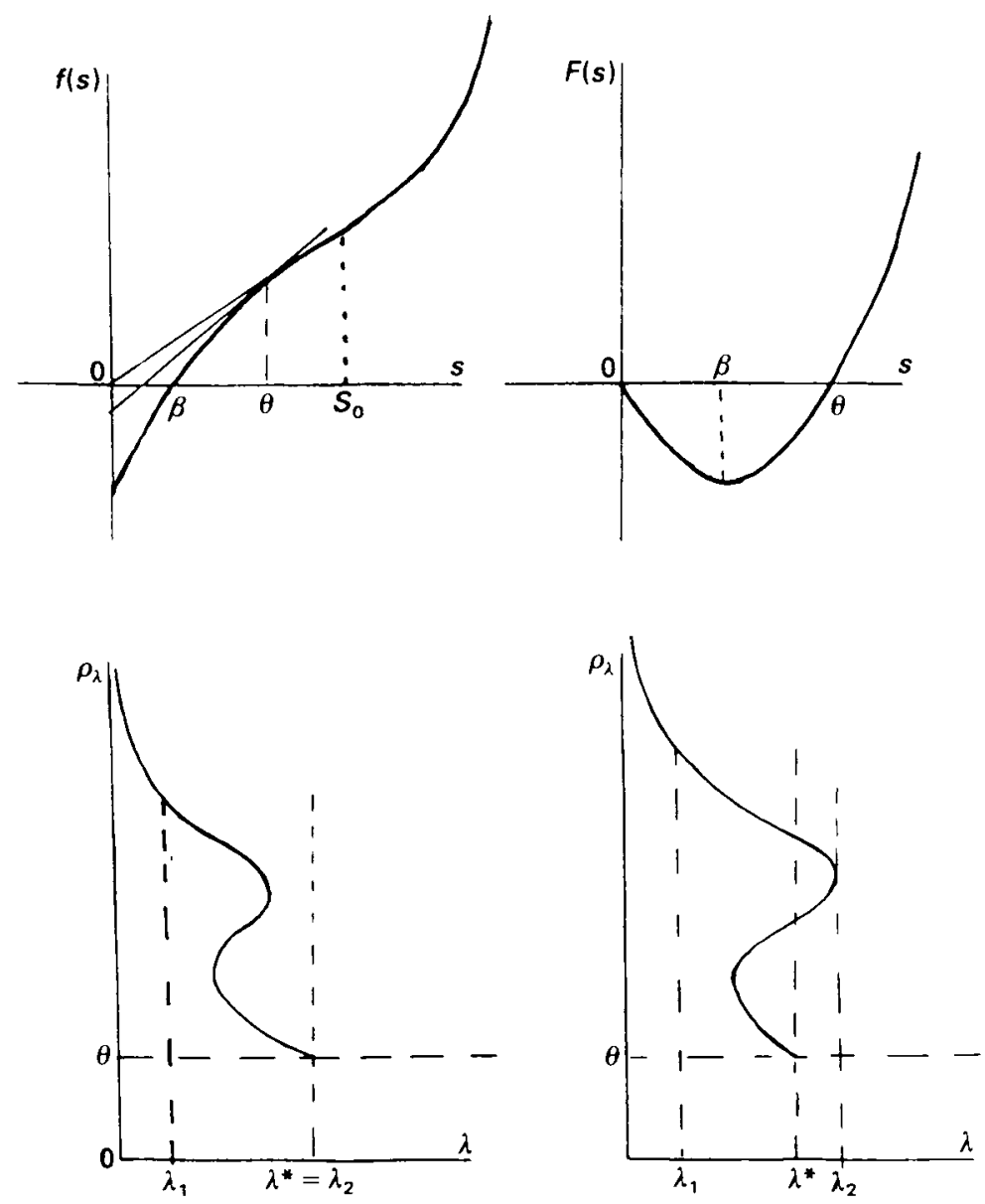

Figure 1.3. Bifurcation diagram (Theorem 1.1, Case (C)).

Remark 1.5. The above results on positive solutions are in contrast to the case of positone problems (see [1]-[3]) where concavity (not convexity) guaranteed uniqueness while convexity (not concavity) allowed multiplicity to be a possibility.

Remark 1.6. Our results are related to the fact that the Hessian of the variational functional corresponding to

$$
-u^{\prime \prime}(x)=f(u(x))-f(0) ; \quad 0<x<1
$$

is non-singular at a positive solution. However, our assumptions are quite general and as such are not enough to guarantee the above.

Remark 1.7. Unlike the case of positone problems where $f(0)>0$, here, in general, it seems rather difficult to apply the method of sub-super solutions to track down the positive solutions. This is because $f(0)<0$ makes $\psi \equiv 0$ a supersolution and not a subsolution as in the case of positone problems. In fact, 
for many of these solutions we suspect that it will not be possible to use this method. However, see [4], where boundary value problems of the type

$$
\begin{aligned}
-\Delta u & =\lambda\left(u-u^{3}\right)-\varepsilon ; & & x \in \Omega \subset R^{n}, \\
u & =0 ; & & x \in \partial \Omega,
\end{aligned}
$$

were considered, and existence of positive solutions for a certain range of $\lambda$ and $\varepsilon>0$ small enough were derived, using sub-super solutions via the anti-maximum principle due to P. Clement and L. A. Peletier.

Remark 1.8. See [5], where (1.1)-(1.3) are considered when $f^{\prime \prime}(u)<0$ and when $f$ satisfies certain additional hypotheses which are not easy to verify in general. The authors prove that, at most, there are only two positive solutions for any $\lambda$.

We shall prove our results in Section 2. In Section 3 we give an example satisfying the hypotheses of Theorem 1.1. In particular, we discuss Case (C), as construction of examples for Cases (A) and (B) is much easier.

\section{Proofs of results}

Proof of Theorem 1.1. On multiplying (1.1) by $u^{\prime}(x)$ and integrating, we obtain

$$
-\left[u^{\prime}(x)\right]^{2} / 2=\lambda F(u(x))+C .
$$

Since we are dealing with positive solutions, $u(x)$ has to be symmetric with respect to $x=1 / 2$ and $u^{\prime}(x)>0$ for $x \in\left(0, \frac{1}{2}\right)$, see Figure 2.1

In fact, if $\rho=\operatorname{Sup}_{x \in(0,1)} u(x)$ then $u\left(\frac{1}{2}\right)=\rho, \rho \geqq \theta$, and substituting $x=\frac{1}{2}$ in (2.1) we obtain

$$
u^{\prime}(x)=\sqrt{2 \lambda[F(\rho)-F(u)]} ; \quad x \in\left[0, \frac{1}{2}\right] .
$$

Now integrating (2.2) on $[0, x]$ and using (1.2), we obtain

$$
\int_{0}^{u(x)} \frac{d u}{\sqrt{F(\rho)-F(u)}}=\sqrt{2 \lambda} x ; \quad x \in\left[0, \frac{1}{2}\right]
$$

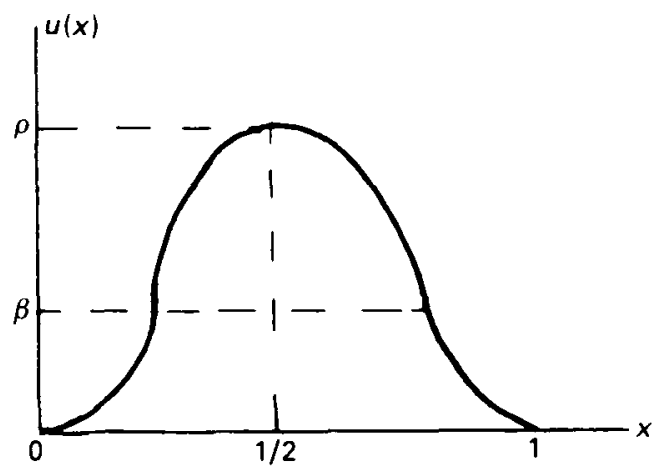

Figure 2.1 


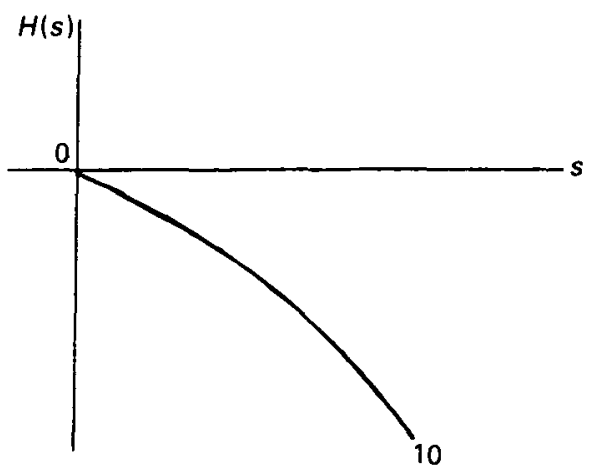

Figure 2.2

and hence substituting $x=\frac{1}{2}$ in (2.3) we have

$$
\sqrt{\lambda}=\sqrt{2} \int_{0}^{\rho} \frac{d u}{\sqrt{F(\rho)-F(u)}}:=G(\rho) .
$$

Now for positive solutions $\rho$ must be in $[\theta, \infty)$. In fact, for given $\lambda$ such that there exists a $\rho \in[\theta, \infty)$ for which $G(\rho)=\sqrt{\lambda}$, it follows that (1.1)-(1.2) have a positive solution $u(x)$ given by (2.3) such that $\operatorname{Sup}_{x \in(0,1)} u(x)=u\left(\frac{1}{2}\right)=\rho$. Further, it follows that $G(\rho)$ is a continuous function and differentiable for $\rho \in(\theta, \infty)$ with

$$
\frac{d}{d \rho} G(\rho)=\sqrt{2} \int_{0}^{1} \frac{H(\rho)-H(\rho V)}{[F(\rho)-F(\rho V)]^{\frac{3}{2}}} d V,
$$

where

$$
H(s)=F(s)-(s / 2) f(s)
$$

Case (A). We will prove that $G^{\prime}(\rho)<0$ and $\lim _{\rho \rightarrow+\infty} G(\rho)=0$. Now $H^{\prime}(s)=$ $\frac{1}{2}\left[f(s)-s f^{\prime}(s)\right]$ and $H^{\prime \prime}(s)=-\left(\frac{1}{2}\right) s f^{\prime \prime}(s)$. But $f(0)<0, f^{\prime \prime}(s)>0$ for $s>0$. Thus $H^{\prime}(s)<0$ for $s>0$, see Figure 2.2.

Hence $H(\rho)-H(\rho v)<0$ for $v \in[0,1]$, and consequently $G^{\prime}(\rho)<0$. Next, note that for $\rho \in(\theta, \infty)$,

$$
G(\rho)=\sqrt{2} \frac{\rho}{\sqrt{F(\rho)}} \int_{0}^{1} \frac{d v}{\sqrt{1-[F(\rho v) / F(\rho)]}} .
$$

Let $L(v):=F(\rho v) / F(\rho)$. Then $L(0)=0, L^{\prime}(v)=(f(\rho v) \rho) / F(\rho)$ and $L^{\prime \prime}(v)=$ $\left(f^{\prime}(\rho v) \rho^{2}\right) / F(\rho)$. But $f(s)<0$ for $s \in(0, \beta), f(s)>0$ for $s>\beta$ and $f^{\prime}(s)>0$ for $s>0$. Hence for a given $\rho \in(\theta, \infty), L(v)$ takes the shape shown in Figure 2.3.

Hence, clearly, $L(v) \leqq v$ for $v \in[0,1]$. Consequently from (2.7)

$$
G(\rho) \leqq \sqrt{2} \frac{\rho}{\sqrt{F(\rho)}} \int_{0}^{1} \frac{d v}{\sqrt{1-v}}=2 \sqrt{2} \rho / \sqrt{F(\rho)} .
$$




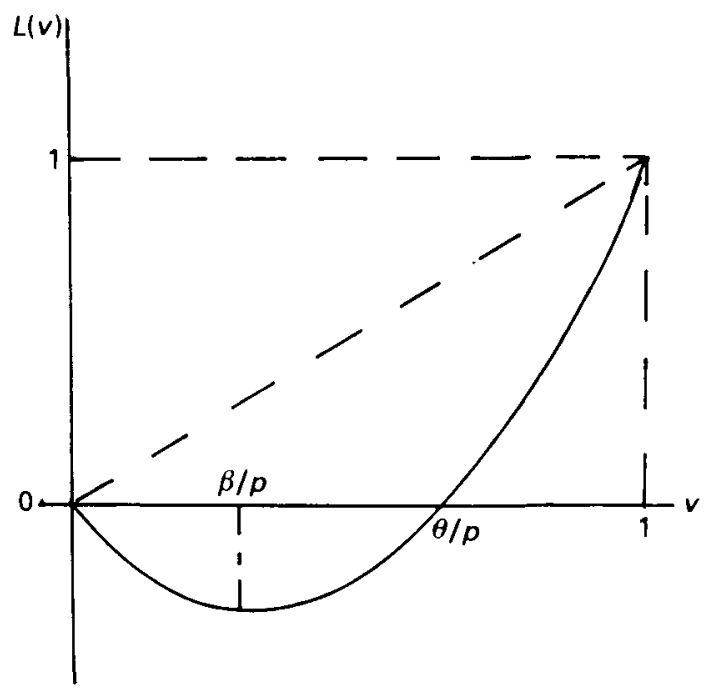

Figure 2.3

But since $\lim _{s \rightarrow+\infty} f(s) / s=+\infty$, we have $\lim _{\rho \rightarrow+\infty} \rho^{2} / F(\rho)=\lim _{\rho \rightarrow+\infty} 2 \rho / f(\rho)=0$, and hence $\lim _{\rho \rightarrow+\infty} G(\rho)=0$. This completes the proof of Case (A).

Case (B). Note that $f(0)<0, f^{\prime \prime}(s)<0$ for $s>0, f(\theta)<\theta f^{\prime}(\theta)$ and $\lim _{s \rightarrow+\infty} f(s)=$ $M$. Thus $H^{\prime \prime}(s)>0$ for $s>0, H^{\prime}(s)<0$ for $s \in[0, \theta]$ and $\lim _{s \rightarrow+\infty} H^{\prime}(s)=(M / 2)>0$.

Hence there exists $\delta, \gamma$ such that $\theta<\delta<\gamma, H^{\prime}(\delta)=0$ and $H(\gamma)=0$, see Figure 2.4. Consequently, $G^{\prime}(\rho)<0$ for $\rho \in[0, \delta]$ and $G^{\prime}(\rho)>0$ for $\rho \geqq \gamma$ and, in order to complete this part of the proof it remains to prove that $\lim _{\rho \rightarrow+\infty} G(\rho)=+\infty$.

Now $L(v)=F(\rho v) / F(\rho) \geqq[f(0) \rho v] / F(\rho)$, since $F^{\prime \prime}=f^{\prime}>0$. Hence from (2.7)

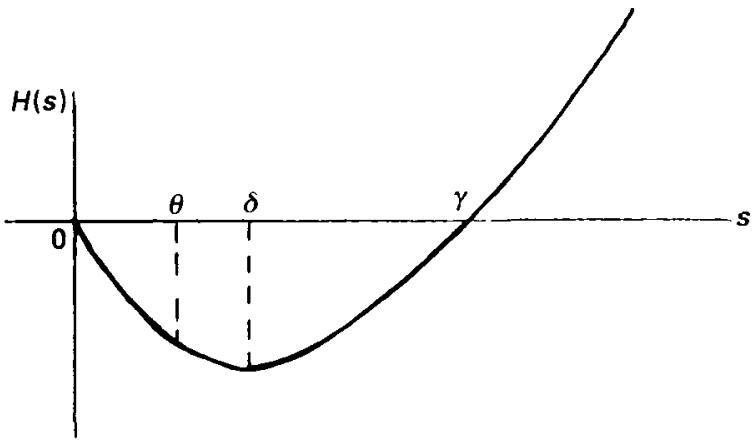

Figure 2.4 
we have

$$
\begin{aligned}
G(\rho) & \geqq \sqrt{2}(\rho / \sqrt{F(\rho)}) \int_{0}^{1} \frac{d v}{\sqrt{1-[(f(0) \rho v) / F(\rho)]}} \\
& =-\sqrt{2}(\sqrt{F(\rho)} / f(0)) \int_{0}^{-(f(0) \rho) / F(\rho)} \frac{d \omega}{\sqrt{1+\omega}},
\end{aligned}
$$

where $w=-(f(0) \rho v) / F(\rho)$. Hence

$$
\begin{aligned}
G(\rho) & \geqq-2 \sqrt{2}(\sqrt{F(\rho)} / f(0))\left\{[1-(f(0) \rho) / F(\rho)]^{\frac{1}{2}}-1\right\} \\
& =2 \sqrt{2}(\rho / \sqrt{F(\rho)})\left\{[1-(f(0) \rho) / F(\rho)]^{\frac{1}{2}}+1\right\}^{-\frac{1}{2}} .
\end{aligned}
$$

But $\lim _{\rho \rightarrow+\infty} \rho / F(\rho)=\frac{1}{M} \quad$ while $\quad \lim _{\rho \rightarrow+\infty} \rho^{2} / F(\rho)=\lim _{\rho \rightarrow+\infty} 2 \rho / f(\rho)=+\infty$. Hence $\lim _{\rho \rightarrow+\infty} G(\rho)=+\infty$ and Case (B) is proved.

Case (C). The hypotheses imply that $H(s)$ takes the form shown in Figure 2.5, which shows $\sigma_{1}, \sigma_{2}, \sigma_{3}$ and $\sigma_{4}$.

Hence $H(\rho)-H(\rho v)<0$ for $v \in[0,1)$ when $\rho \in\left[\theta, \sigma_{1}\right]$ and when $\rho>\sigma_{4}$, while $H(\rho)-H(\rho v)>0$ for $v \in[0,1)$ when $\rho \in\left(\sigma_{2}, \sigma_{3}\right]$. Consequently $G^{\prime}(\rho)<0$ for $\rho \in\left[\theta, \sigma_{1}\right]$ and for $\rho \geqq \sigma_{4}$, while $G^{\prime}(\rho)>0$ for $\rho \in\left[\sigma_{2}, \sigma_{3}\right]$. Now to complete the proof of Theorem 1.1 it remains to prove that $\lim _{\rho \rightarrow+\infty} G(\rho)=0$, which follows by arguments identical to those used in the proof of Case (A).

Proof of Lemma 1.2. First we note that $(1-\sqrt{-F(s)}) \geqq(1 / \sqrt{-F(\beta)})$ for

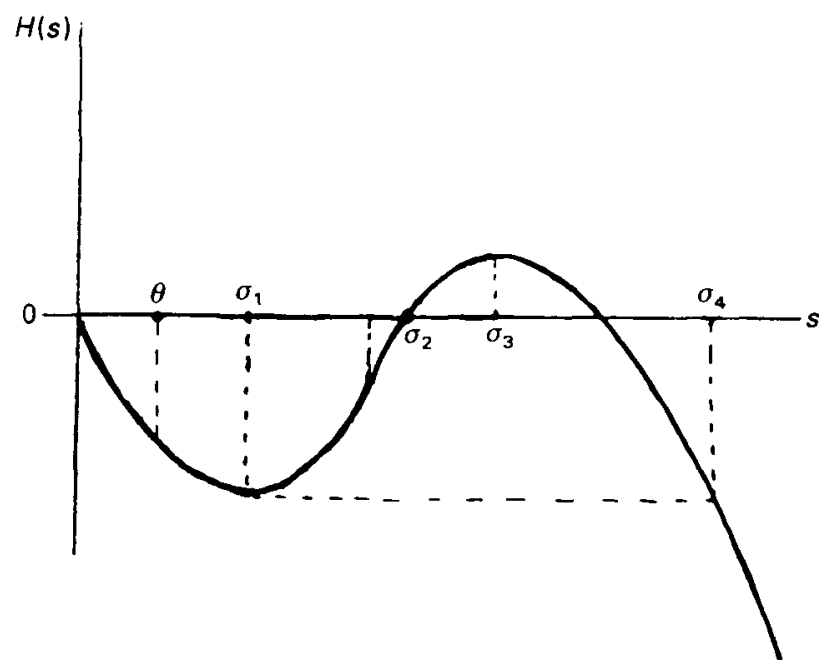

Figure 2.5 


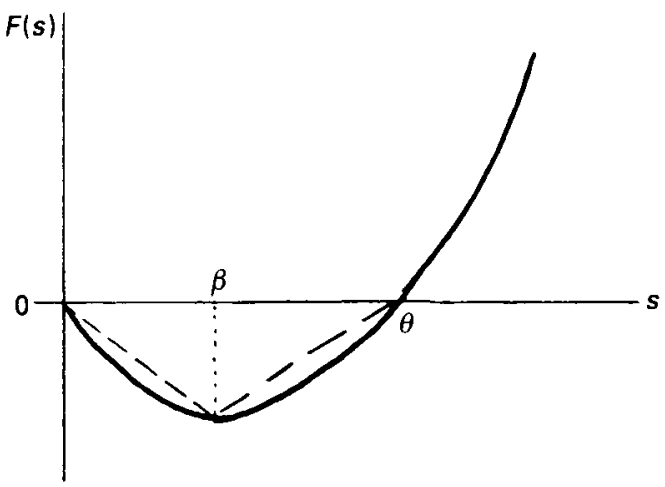

Figure 2.6

$s \in(0, \theta)$, and since $F^{\prime \prime}(s)=f^{\prime}(s)>0$ for $s>0$ we have

$$
-F(s) \geqq\left\{\begin{array}{l}
-(F(\beta) / \beta) s \text { for } 0 \leqq s \leqq \beta, \\
-[F(\beta) /(\theta-\beta)](\theta-s) \text { for } \beta<s \leqq \theta .
\end{array}\right.
$$

(See Fig. 2.6.)

Thus

$$
(1 / \sqrt{-F(s)}) \leqq\left\{\begin{array}{l}
\sqrt{\beta /(-F(\beta) s)} \text { for } 0 \leqq s \leqq \beta \\
\sqrt{(\theta-\beta) /[-F(\beta)(\theta-s)]} \text { for } \beta<s \leqq
\end{array}\right.
$$

Hence

$$
G(\theta)=\sqrt{2} \int_{0}^{\theta} \frac{d s}{\sqrt{-F(s)}} \geqq \sqrt{2} \theta / \sqrt{-F(\beta)},
$$

and

$$
\begin{aligned}
G(\theta) & =\sqrt{2} \int_{0}^{\beta} \frac{d s}{\sqrt{-F(s)}}+\sqrt{2} \int_{\beta}^{\theta} \frac{d s}{\sqrt{-F(s)}} \\
& \leqq \sqrt{2} \sqrt{\beta /(-F(\beta))} 2 \sqrt{\beta}+\sqrt{2} \sqrt{(\theta-\beta) /(-F(\beta))} 2 \sqrt{\theta-\beta} \\
& =2 \sqrt{2} \theta / \sqrt{-F(\beta)} .
\end{aligned}
$$

From (2.8) and (2.9) it easily follows that $2 \alpha \leqq \lambda^{*}=[G(\theta)]^{2} \leqq 8 \alpha$, where $\alpha=\theta^{2} /[-F(\beta)]$, and Lemma 1.2 is proved. Note here that for $\lambda=\lambda^{*}$ the minimal solution $\underline{u}(x)$ has supremum norm $\rho=\theta$ and hence $\underline{u}^{\prime}(0)=0$, see Figure 2.7.

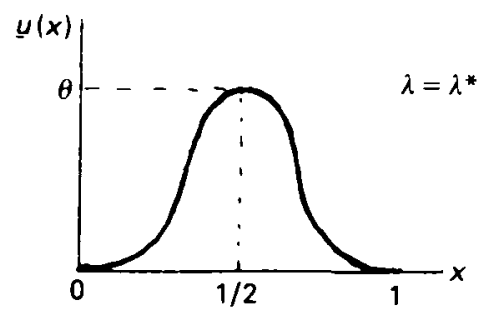

Figure 2.7 


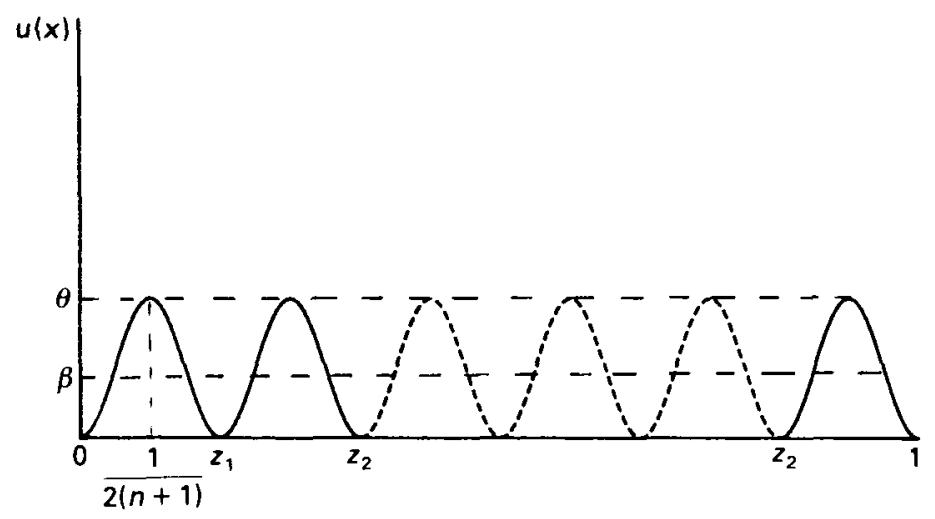

Figure 2.8

Proof of Lemma 1.3. If $u_{2}\left(\frac{1}{2}\right)=\rho_{2}>u_{1}\left(\frac{1}{2}\right)=\rho_{1}$, then at any point $x_{o} \in\left(0, \frac{1}{2}\right)$ where $u_{1}\left(x_{0}\right)=u_{2}\left(x_{0}\right)$, from (2.2) we must have $u_{2}^{\prime}\left(x_{0}\right)>u_{1}^{\prime}\left(x_{0}\right)$. Hence, there can be at most one such point. But from (2.2) we also have $u_{2}^{\prime}(0)>u_{1}^{\prime}(0)$. Thus, no such point exists.

Proof of Theorem 1.4. First we note that in order to study a non-negative solution with $n$ interior zeros, due to the symmetry we need to study the solution only in the interval $[0,1 /(2(n+1))]$, see Figure 2.8 .

From (2.1) we have for $x \in[0,1 /(2(n+1))]$,

$$
u^{\prime}(x)=\sqrt{2 \lambda[F(\rho)-F(u)]},
$$

where $\rho=\sup _{x \in[0,1]} u(x)=u(1 /(2(n+1)))$. But since we must have $u^{\prime}(0)=0$, $F(\rho)=0$ and hence $\rho=\theta$. Now integrating (2.10) on $[0, x]$, we obtain

$$
\int_{0}^{u(x)} \frac{d u}{\sqrt{-F(u)}}=\sqrt{2 \lambda} x ; \quad x \in[0,1 /(2(n+1))],
$$

and hence substituting $x=1 /(2(n+1))$ in (2.11) we must have

$$
\sqrt{\lambda}=(n+1) \sqrt{2} \int_{0}^{\theta} \frac{d u}{\sqrt{-F(u)}}=(n+1) \sqrt{\lambda^{*}} .
$$

Thus, in order to have a non-negative solution $u(x)$ to (1.1)-(1.2) with $n$ interior zeros, $\lambda$ must equal $(n+1)^{2} \lambda^{*}$ and $\operatorname{Sup}_{x \in[0,1]} u(x)=\theta$. In fact, given $\lambda=(n+1)^{2} \lambda^{*}$ it follows that (1.1)-(1.2) have a unique non-negative solution $u(x)$ with $n$ interior zeros given by $(2.11)$ with $\operatorname{Sup}_{x \in[0,1]} u(x)=u(1 /(2(n+1)))=\theta$.

\section{An example}

This example illustrates Theorem 1.1, Case (C). Consider

$$
f(s)=s^{3}-a s^{2}+b s-c,
$$


where $a>0, b>0, c>0$ and they satisfy

$$
\begin{gathered}
b>(32 / 81) a^{2}, \\
a^{3}>54 c .
\end{gathered}
$$

Clearly $f(0)<0$. Further

$$
\begin{aligned}
f^{\prime}(s) & =3 s^{2}-2 a s+b \\
& =3\left\{[s-(a / 3)]^{2}+(b / 3)-\left(a^{2} / 9\right)\right\} .
\end{aligned}
$$

But (3.2) implies that $(b / 3)>\left(a^{2} / 9\right)$. Hence $f^{\prime}(s)>0$ for $s>0$ and since $\lim _{s \rightarrow+\infty} f(s)=+\infty$, there exists a unique $\beta>0$ such that $f(\beta)=0$.

Next $F(s)=s g(s)$, where

$$
g(s)=\left(s^{3} / 4\right)-\left(a s^{2} / 3\right)+(b s / 2)-c,
$$

and

$$
\begin{aligned}
g^{\prime}(s) & =\left(3 s^{2} / 4\right)-(2 a s / 3)+(b / 2) \\
& =(3 / 4)\left\{[s-(4 a / 9)]^{2}+(2 b / 3)-\left(16 a^{2} / 81\right)\right\} .
\end{aligned}
$$

Once again (3.2) implies that $(2 b / 3)>\left(16 a^{2} / 81\right)$ and hence $g^{\prime}(s)>0$ for $s \geqq 0$. But $g(0)<0$ and $\lim _{s \rightarrow+\infty} g(s)=+\infty$. Hence there exists a unique $\theta>\beta$ such that $F(\theta)=g(\theta)=0$.

Now note that

$$
f^{\prime \prime}(s)=6 s-2 a .
$$

Clearly there exists $s_{0}=(a / 3)$ such that $f^{\prime \prime}(s)<0$ for $s \in\left(0, s_{0}\right)$ and $f^{\prime \prime}(s)>0$ for $s>s_{0}$. Also (3.2), (3.3) imply that

$$
\begin{aligned}
F(a / 3) & =(a / 3)\left\{\left(a^{3} / 108\right)-\left(a^{3} / 27\right)+(a b / 6)-c\right\} \\
& =(a / 3)\left\{(a b / 6)-c-\left(a^{3} / 36\right)\right\} \\
& \geqq(a / 3)\left\{\left(32 a^{3} / 486\right)-\left(a^{3} / 54\right)-\left(a^{3} / 36\right)\right\} \\
& =\left(19 a^{4} / 2916\right)>0 .
\end{aligned}
$$

Hence $\theta<s_{0}=(a / 3)$.

Next consider

$$
f(\theta)-\theta f^{\prime}(\theta)=-2 \theta^{3}+a \theta^{2}-c .
$$

Now $g(\theta)=0$ and thus substituting for $c$ we have

$$
\begin{aligned}
f(\theta)-\theta f^{\prime}(\theta) & =\left(-9 \theta^{3} / 4\right)+\left(4 a \theta^{2} / 3\right)-(b \theta / 2) \\
& =(-9 \theta / 4)\left\{[\theta-(8 a / 27)]^{2}+(6 b / 27)-\left[64 a^{2} /(27)^{2}\right]\right\} .
\end{aligned}
$$

Hence, using (3.2), we have $f(\theta)-\theta f^{\prime}(\theta)<0$.

Finally, the hypotheses $\lim _{s \rightarrow+\infty}(f(s) / s)=+\infty$ and $\lim _{s \rightarrow+\infty} f(s)-\lim _{s \rightarrow+\infty} s f^{\prime}(s)<0$ are easily seen to be satisfied, and hence it remains to prove the existence of a 
number $\sigma>\theta$ such that $H(\sigma)>0$. Inequality (3.3) implies that

$$
\begin{aligned}
H(a / 3) & =\left(-a^{4} / 324\right)+\left(a^{4} / 162\right)-(a c / 6) \\
& =\left\{\left[a^{4}-54 a c\right] / 324\right\}>0,
\end{aligned}
$$

and $(a / 3)>\theta$. Hence the result.

\section{References}

1 K. J. Brown, M. M. A. Ibrahim and R. Shivaji. S-shaped bifurcation curves. Nonlinear Anal. 5 (1981), 475-486.

2 R. Shivaji. Uniqueness results for a class of positone problems. Nonlinear Anal. 2 (1983), 223-230.

3 T. W. Laetsch. The number of solutions of a nonlinear two point boundary value problem. Indiana Univ. Math. J. 20 (1970/71), 1-13.

4 K. J. Brown and R. Shivaji. Simple proofs of some results in perturbed bifurcation theory. Proc. Roy. Soc. Edinburgh Sect. A 93 (1982), 71-82.

5 P. Brunovsky and S. N. Chow. Generic properties of stationary state solutions of reactiondiffusion equations. J. Differential Equations 53 (1984), 1-23.

(Issued 27 April 1988) 